\title{
Charge Transport Through a Single Molecular Wire Based on Linear Multimetal Complexes: A Non-Equilibrium Green's Function Approach
}

\author{
Liang-Yan Hsu, Qian-Rui Huang, and Bih-Yaw Jin* \\ Department of Chemistry and Center of Theoretical Sciences, National Taiwan University, Taipei, \\ Taiwan, ROC
}

Received: March 5, 2008; Revised Manuscript Received: April 25, 2008

\begin{abstract}
The Non-Equilibrium Green's Function method and the extended Hückel theory (the NEGF-EHT) are employed to study transmission functions and conductance of linear trimetal complexes, $\mathrm{M}_{3}\left(\mu_{3} \text {-dpa }\right)_{4}(\mathrm{NCS})_{2}(\mathrm{dpa}=$ 2, 2'-dipyridylamide), $\mathrm{M}=\mathrm{Co}, \mathrm{Ni}$, and $\mathrm{Cr}$. The trend and orders of magnitude of the resulting conductance based on the independent electron approach are in good agreement with the experimental observations. Moreover, the effects of ligands on the conduction pathways of molecular wires are investigated comparatively by studying a family of hypothetical complexes coordinated with different ligands. We have found that one of the molecular orbitals consisting of the central $\mathrm{d}_{z^{2}}$ orbitals in metal strings plays a major role in electron transport of linear trimetal complexes.
\end{abstract}

During the past decade, the investigations of single molecular electronics have received much attention both experimentally and theoretically because of its potential application to nanoelectronic devices. ${ }^{1,2}$ Besides the most extensively studied BDT molecular wires, ${ }^{3}$ charge transport through Au wires, ${ }^{4,5}$ hydrogen molecules, ${ }^{6}$ and small organic molecules ${ }^{7-9}$ has also been investigated by many groups. In addition to the types of molecular wires mentioned above, chemists have also synthesized various organic and inorganic compounds as potential candidates of molecular wires. Among them, the extended metal atom chain (EMAC) complexes of the type $\mathrm{M}_{3}\left(\mu_{3} \text {-dpa }\right)_{4}(\mathrm{X})_{2}$ (dpa $=2,2$-dipyridylamide, $X=\mathrm{Cl}$, NCS) (see Figure 1) have been extensively studied in several different laboratories. ${ }^{10,11}$

EMAC molecules are not only model systems for better understanding of metal-metal bonding, ${ }^{12-17}$ but also promising candidates as single molecular wires or devices. This is because the EMAC compounds are a nanoscale version of ordinary everyday electrical wires. Measurements of the $I-V$ characteristics and conductance of linear multimetal complexes containing up to seven metal atoms were carried out recently. ${ }^{11}$ An interesting dependence of conductance on the type and number of metal atoms along the metal string has been observed and rationalized on the basis of simple bonding arguments. However, to the best of our knowledge, no theoretical investigation has been performed to attain detailed transport mechanisms of the molecular wires consisting of EMAC complexes.

In this article, we applied the NEGF method with the phasebreaking processes considered by using the Landauer-Büttiker formalism. ${ }^{18-20}$ The phase-breaking processes are mainly caused by the self-energy of molecular vibration and electrodes. The selfenergy contributed by molecular vibration is assumed to be proportional to the density of single-particle states. ${ }^{19,20}$ On the other hand, the self-energy due to coupling to the electrodes is obtained from the calculation of surface Green's function..$^{21,22}$ We have adopted the extended Hückel Hamiltonian for the calculation of molecular wires based on two different parameter sets. The first one is the parameter set implemented in the HuckelIV 2.0, ${ }^{23}$ while the second one is from the Yaehmop, ${ }^{24}$ in which the orbital

\footnotetext{
*byjin@ntu.edu.tw.
}

asymmetry parameter is used to take care of the counterintuitive orbital mixing. ${ }^{25}$ We have assumed the EMAC molecule is connected to the $\mathrm{Au}$ electrode through the $\mathrm{S}-\mathrm{Au}(111)$ junction at the hollow position. The distance between the $\mathrm{S}$ atom and the $\mathrm{Au}$ surface is chosen to be $1.905 \AA$ as determined by DFT optimization in the previous work. ${ }^{26}$ The Fermi level of the Au electrode is chosen to be $-9.54 \mathrm{eV}^{27,28}$ in order to get the best agreement with experiments.

The calculated transmission functions and conductance of linear trimetal complexes, $\mathrm{M}_{3}\left(\mu_{3} \text {-dpa }\right)_{4}(\mathrm{NCS})_{2}, \mathrm{M}=\mathrm{Co}, \mathrm{Cr}$, $\mathrm{Ni}$, are shown in Figure 2 and Table 1. Two sets of experimental data are presented in Table 1: the data (exp I) in the first column are obtained from the c-AFM measurement in vacuum; while the other set of data (exp II) are based on the STM break junction technique in solution. ${ }^{11}$ The relative trend of conductances of these three linear trimetal complexes is in good agreement with experimental observations measured by both methods: trichromium $>$ tricobalt $>$ trinickel. Moreover, the order of magnitude of our theoretical results (methods I and II in Table 1) using two different parameter sets seems to be very close to the data from the c-AFM measurement. This is reasonable since our calculations are performed on a single molecular junction in a vacuum. Figure 2 shows that the major peak in the transmission spectrum of the tricobalt complexes is located at $-10.18 \mathrm{eV}$. The probability amplitude of the molecular orbital responsible for this peak is mainly distributed on the central metal strings (out-of-phase linear combination of three $\mathrm{d}_{z^{2}}$ orbitals) and the sulfur atoms $\left(\mathrm{p}_{z}\right)$, indicating that the most important conduction pathway is through the central metal string, with little contribution from the peripheral ligands. Similar to the tricobalt complexes, the major peak in the transmission spectrum of the trichromium complexes is also originating from the central $\mathrm{d}_{z^{2}}$ orbitals with the energy slightly closer to the Fermi level $\left(E_{\mathrm{F}}\right)$ as shown in Figure 2, leading to a higher conductance for the molecular wire. As for the trinickel complexes, the most important resonant state relevant for the conduction consists mainly of the $\mathrm{d}_{z^{2}}$ orbital with a much lower energy below $-11 \mathrm{eV}$ (not exhibited in Figure 2). Note that the existence of resonant states near the Fermi level may not guarantee a large contribution to the overall conductance if their 


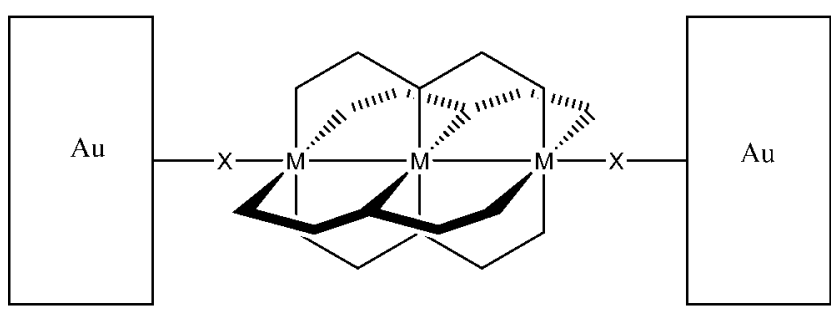<smiles>CCCC(C)CCC</smiles><smiles>c1ccc(Nc2ccccn2)nc1</smiles>

Figure 1. Diagram of linear trimetal complexes $M_{3} L_{4} X_{2}$. $M=C o ; L=\mu_{3}$-dpa; $X=N C S$.

transmission amplitudes are small. For instance, in the cases of the tricobalt and trinickel complexes, even though there are several resonant states (tricobalt complexes: $-9.43 \mathrm{eV}$; trinickel complexes: $-9.74 \mathrm{eV}$ ) mainly concentrated on the surrounding ligands, near Fermi level, they have only an insignificant contribution to the overall conductance. We have also examined the influence of the location of the Fermi level on the relative magnitudes of conductance for these metal complexes. As an example, at $E_{\mathrm{F}}=-9.74 \mathrm{eV}$, the conductance becomes 10.6, 48, and $10400 \mathrm{pS}$ for $\mathrm{Ni}, \mathrm{Co}$, and $\mathrm{Cr}$ systems, respectively. Consistent with the transmission spectrum in Figure 2, a change of the Fermi level within the energy window between -9.9 and $-9.3 \mathrm{eV}$ does not affect the relative trend of three metal complexes.

In order to figure out the relationship between the MOs and the transmission function, we derived a useful formula to explain which $\mathrm{MO}$ can provide larger contribution to conduction

$$
T(E) \approx \frac{4 \alpha^{2} \sum_{\mu \nu}\left|C_{\mu \mathrm{n}}^{(0)}\right|{ }^{2}\left|C_{\nu \mathrm{n}}^{(0)}\right|^{2}}{\left(E-\varepsilon_{n}^{(0)}\right)^{2}+\alpha^{2}\left(\sum_{\mu}\left|C_{\mu \mathrm{n}}^{(0)}\right|^{2}+\sum_{\nu}\left|C_{\nu \mathrm{n}}^{(0)}\right|^{2}\right)^{2}}
$$

where $\mu$ and $v$ denote the atomic orbitals of junction atoms adjacent to the left and right electrodes. $\alpha$ in eq 1 is the imaginary part of self-energy. This formula is based on the approximation of the first-order perturbation, the nearestneighbor couplings between electrodes and a molecular wire, and the neglect of off-diagonal terms of self-energy. It is noted that the off-diagonal terms of self-energy could have nontrivial influence on the transmission function in some special cases. ${ }^{29}$

This formula can be used to estimate the height and halfwidth of the transmission function in preliminary analysis. It is evident that the Cauchy-Schwarz inequality, $4 \Sigma_{\mu v}\left|C_{1 n}^{(0)}\right|\left|C_{N n}{ }^{(0)}\right| \leq$ $\Sigma_{\mu}\left|C_{l n}^{(0)}\right|^{2}+\Sigma_{\nu}\left|C_{N n}{ }^{(0)}\right|^{2}$, ensures that $T(E) \leq 1$. The height of transmission function becomes the maximum when $\left|C_{\mu n}^{(0)}\right|$ and $\left|C_{v n}^{(0)}\right|$ are equal. When the sum of $\Sigma_{\mu}\left|C_{\mu n}^{(0)}\right|$ and $\Sigma_{v}\left|C_{v n}^{(0)}\right|$ are larger, the half-width of the transmission function becomes larger. The squares of $\mathrm{MO}$ coefficients on sulfur atoms in these three types of linear trimetal complexes are listed in Table 2. On the basis of the discussion above, the peak at $-10.18 \mathrm{eV}(-9.90)$ in the transmission spectrum of tricobalt (trichromium) complexes is the most important since the magnitudes of the corresponding MO coefficients on the sulfur atoms to the left and right electrodes are the largest and close to each other in the range of $-9 \mathrm{eV}$ to $-11 \mathrm{eV}$. Besides, this method can also explain why the peak at $-9.43 \mathrm{eV}$ of tricobalt complexes and the peak at $-9.74 \mathrm{eV}$ of trinickel complexes provide little contribution to conduction.

In order to understand the mechanism and to clarify the detailed pathways of conduction in the trimetal complexes, we performed calculations on the hypothetical systems derived from our model compound, $\mathrm{Co}_{3}\left(\mu_{3} \text {-dpa }\right)_{4}(\mathrm{NCS})_{2}$. We construct two

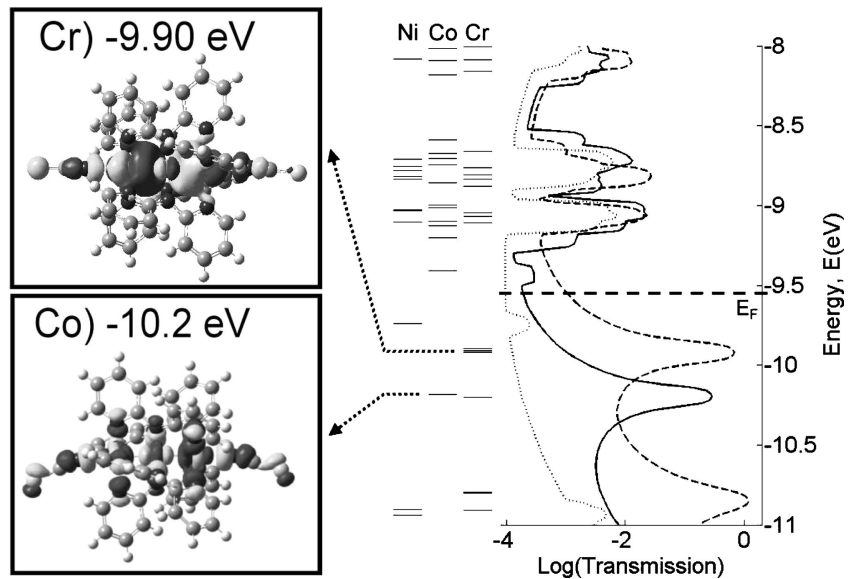

Figure 2. The transmission functions, the MO energy diagrams, and some typical MO diagrams of the EMAC molecules. The solid line: $\left[\mathrm{Co}_{3}\left(\mu_{3} \text {-dpa }\right)_{4}(\mathrm{NCS})_{2}\right]$; the dotted line: $\left[\mathrm{Ni}_{3}\left(\mu_{3} \text {-dpa }\right)_{4}(\mathrm{NCS})_{2}\right]$; the dashed line: $\left[\mathrm{Cr}_{3}\left(\mu_{3} \text {-dpa }\right)_{4}(\mathrm{NCS})_{2}\right]$.

TABLE 1: Experimental and Theoretical Conductance of

\begin{tabular}{|c|c|c|c|c|}
\hline \multirow[b]{2}{*}{ metal } & \multicolumn{2}{|c|}{ experimental values $\left(10^{-9} S\right)$} & \multicolumn{2}{|c|}{ theoretical calculation ${ }^{a}\left(10^{-9} S\right)$} \\
\hline & $\exp \mathrm{I}^{b}$ & $\exp \mathrm{II}^{c}$ & method $\mathrm{I}^{d}$ & method $\mathrm{II}^{e}$ \\
\hline Co & 21 & 530 & 20 & 22 \\
\hline $\mathrm{Ni}$ & 5.8 & 290 & 8.0 & 9.4 \\
\hline $\mathrm{Cr}$ & 370 & 1110 & 200 & 320 \\
\hline
\end{tabular}
Linear Trimetal Complexes

${ }^{a}$ The bias is equal to $0.5 \mathrm{~V}$. ${ }^{b}$ See ref 11 ; the experiment is performed by c-AFM. ${ }^{c}$ See ref 11 ; the experiment is performed by STM. ${ }^{d}$ The calculated results is obtained by Ḧ uckel IV 2.0 which is developed by Purdue's group..$^{23}{ }^{e}$ The Hamiltonian is obtained from Yaehmop. ${ }^{24}$

hypothetical molecules (Figure 3): Molecule 2 is constructed by using four fully conjugated NCHNCHNs as ligands; while molecule $\mathbf{3}$ is generated by removing all four dipyridylamido ligands. The transmission functions and $I-V$ characteristics are calculated with the same NEGF-EHT approach. The result is shown in Figure 4 together with some representative molecular orbitals.

In Figure 4, molecules 1, 2, and $\mathbf{3}$ have the same characteristics that their resonant states consisting of $\mathrm{d}_{z^{2}}$ orbitals have larger transmission than others. The out-of-phase $\mathrm{d}_{z^{2}}$ orbitals of these three molecules are shown in Figure 4. The shapes of the MOs in Figure 4-1 and Figure 4-2 are very similar, implying that the difference between complex and simple ligands is not a major factor to the conduction pathways in the trimetal complexes. On the other hand, as shown by Figure 4-2 and Figure 4-3, the out-of-phase $\mathrm{d}_{z^{2}}$ orbitals are shifted from -12.3 to $-10.1 \mathrm{eV}$ when peripheral ligands are removed completely. Detailed analysis indicated that this shift is mainly caused by the donor atoms of ligands. Thus, through the change of the donor atoms, the position of the $\mathrm{d}_{z^{2}}$ orbital could be tuned. 
TABLE 2: Square of MO Coefficients of Linear Trimetal Complexes ${ }^{a}$

\begin{tabular}{|c|c|c|c|c|c|c|c|c|}
\hline \multicolumn{3}{|c|}{ cobalt } & \multicolumn{3}{|c|}{ nickel } & \multicolumn{3}{|c|}{ chromium } \\
\hline energy $^{b}$ & left $^{c}$ & right & energy & left & right & energy & left & right \\
\hline-10.18 & 0.05865 & 0.02276 & -10.94 & 0.00072 & 0.00020 & -10.91 & 0.00586 & 0.00024 \\
\hline-9.22 & 0.00005 & 0.00501 & -9.74 & 0.00100 & 0.00054 & -10.80 & 0.00040 & 0.00035 \\
\hline-9.14 & 0.00003 & 0.00122 & -9.10 & 0.00050 & 0.00024 & -10.20 & 0.00357 & 0.00320 \\
\hline-9.11 & 0.00076 & 0.03405 & -9.03 & 0.00007 & 0.00029 & -9.92 & 0.00021 & 0.00439 \\
\hline-9.03 & 0.00058 & 0.00001 & -9.03 & 0.00006 & 0.00000 & -9.91 & 0.00007 & 0.02588 \\
\hline & & & & & & -9.07 & 0.00002 & 0.00657 \\
\hline & & & & & & -9.04 & 0.00002 & 0.00291 \\
\hline
\end{tabular}

${ }^{a}$ The magnitudes of MO coefficients on the sulfur atoms can be used to estimate the intensities and widths for the corresponding resonances in the transmission function. ${ }^{b}$ Molecular orbital energy in eV calculated by Yaehmop. ${ }^{c}$ The square of MO coefficients of the left sulfur atoms attached onto the left electrodes.
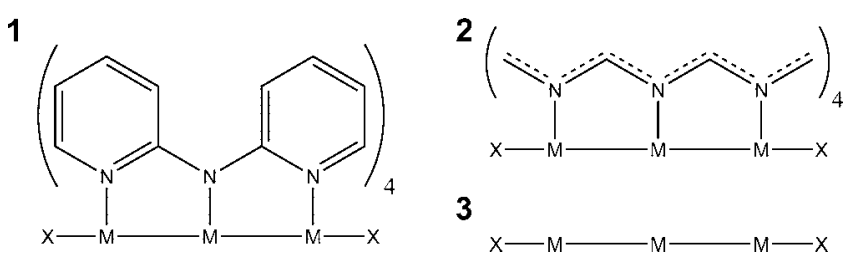

Figure 3. The diagram of hypothetical EMACs with different ligands. $\mathrm{M}=\mathrm{Co}, \mathrm{Ni}, \mathrm{Cr} ; \mathrm{X}=\mathrm{NCS}$.

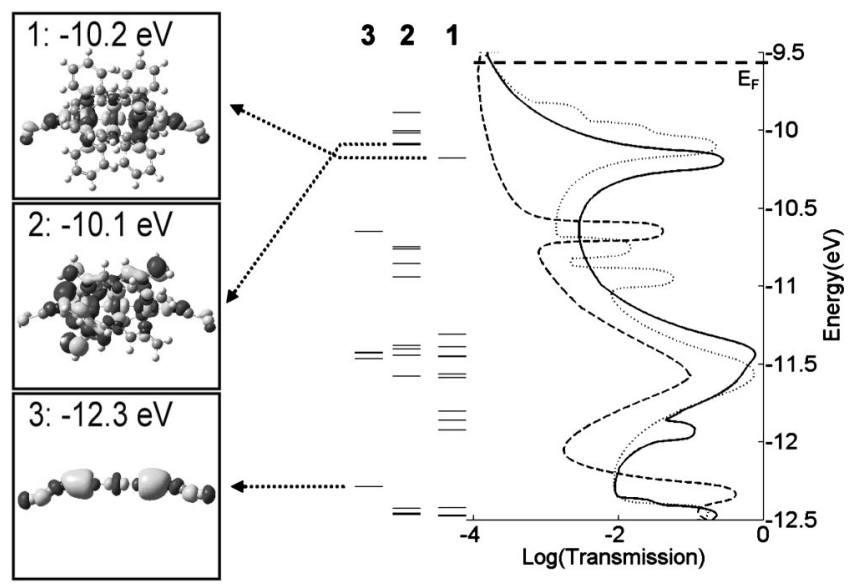

Figure 4. The transmission spectrum, the MO energy diagrams, and some typical MO diagrams of $\mathrm{Co}_{3}\left(\mu_{3}-\mathrm{dpa}\right)_{4}(\mathrm{NCS})_{2}$ and related hypothetical EMACs. (a) The solid line: Molecule 1; (b) the dotted line: Molecule 2; (c) the dashed line: Molecule 3.

According to the calculated transmission function, the MO analysis, and the comparative study of hypothetical systems, we can conclude that one of the $d_{z^{2}}$ molecular orbitals of the central metal string in the linear trimetal complexes plays the most important role in molecular conduction. Besides, this dominant resonant state also has a significant MO amplitude of sulfur atoms, leading to the largest half-width for this peak in the transmission spectrum. It implies that the closer the resonant state consisting of $\mathrm{d}_{z^{2}}$ orbitals is to the Fermi level, the larger the conductance is. This also explains the reason the relative conductance of these three linear trimetal complexes exhibits the trend: trichromium $>$ tricobalt $>$ trinickel. Albeit the success is based on the NEGF-EHT for the trimetal strings, the electron correlation effect is known to play an important role for the EMAC systems. ${ }^{30}$ In order to include the effect of electron correlation on the electron transport properties of the EMAC systems, the NEGF-DFT calculation of the linear trimetal and multimetal complexes is probably necessary.
Acknowledgment. We wish to acknowledge the assistance and support provided by Prof. Shie-Ming Peng and Prof. ChunHsien Chen. We thanks Prof. Datta for providing the HuckelIV 2.0. We also wish to acknowledge the financial support of National Science Council, Taiwan.

Supporting Information Available: The detailed proof of eq 1 . This material is available free of charge via the Internet at http://pubs.acs.org.

\section{References and Notes} 541.

(1) Joachim, C.; Gimzewski, J. K.; Aviram, A. Nature 2000, 408

(2) Tao, N. J. Nat. Nanotechnol. 2006, 1, 173.

(3) Read, M. A.; Zhou, C.; Muller, C. J.; Burgin, T. P.; Tour, J. M. Science 1997, 278, 252. 780 .

(4) Ohnishi, H.; Kondo, Y.; Takayanagi, K. Nature 1998, 395

(5) Yanson, A. I.; Bollinger, G. R.; van den Brom, H. E.; Agrait, N.; van Ruitenbeek, J. M. Nature 1998, 395, 783.

(6) Smit, R. H. M.; Noat, Y.; Untiedt, C.; Lang, N. D.; van Hemert, M. C.; van Ruitenbeek, J. M. Nature 2002, 419, 906.

(7) Xue, Y.; Ratner, M. A. Int. J. Quantum Chem. 2005, 102, 911.

(8) Jang, S. Y.; Reddy, P.; Segalman, R. A.; Majumdar, A. Science 2007, 315, 1568.

(9) Venkataraman, L.; Park, Y. S.; Whalley, A. C.; Nuckolls, C.; Hybertsen, M. S.; Steigerwald, M. L. Nano Lett. 2007, 7, 502.

(10) Chae, D. H.; Berry, J. F.; Jung, S.; Cotton, F. A.; Murillo, C. A.; Yao, Z. Nano Lett. 2006, 2, 165.

(11) Chen, I. P.; Fu, M. D.; Tseng, W. H.; Yu, J. Y.; Wu, S. H.; Ku, C. J.; Chen, C. H.; Peng, S. M. Angew. Chem., Int. Ed. 2006, 45, 5814.

(12) Berry, J. F.; Cotton, F. A.; Lu, T.; Murillo, C. A.; Roberts, B. K.; Wang, X. J. Am. Chem. Soc. 2004, 126, 7082.

(13) Cotton, F. A.; Daniels, L. M.; Murillo, C. A.; Pascual, I. Inorg. Chem. Commum. 1998, 1, 3.

(14) Clérac, R.; Cotton, F. A.; Dunbar, K. R.; Murillo, C. A.; Pascual, I.; Wang, X. Inorg. Chem. 1999, 38, 2655.

(15) Clérac, R.; Cotton, F. A.; Daniels, L. M.; Dunbar, K. R.; Murillo, C. A.; Wang, X. Inorg. Chem. 2001, 40, 1256.

(16) Rohmer, M. M.; Benard, M. J. Am. Chem. Soc. 1998, 120, 9372.

(17) Rohmer, M. M.; Strich, A.; Benard, M.; Malrieu, J. P. J. Am. Chem. Soc. 2001, 123, 9126.

(18) Datta, S. Quantum Transport: Atom to Transistor; Cambridge, 2005.

(19) Datta, S.; Tuan, W.; Song, H.; Reifenberger, R.; Henderson, J. I.; Kubiak, C. P. Phys. Rev. Lett. 1997, 79, 2530.

(20) Datta, S.; Tuan, W.; Song, H.; Reifenberger, R.; Henderson, J. I.; Kubiak, C. P. J. Chem. Phys. 1998, 109, 2874.

(21) Reed, M. A. Molecular Nanoelectronics; American Scientific Publishers: Stevenson Ranch, CA, 2003.

(22) Morkoc, H. Advanced Semiconductors and Organic Nanotechniques; Academic: London, 2003. 
(23) Zahid, F. Huckel-IV 2.0 Source Code Download; https://www. nanohub.org/resources/.

(24) Lamdrum,G. A. ; Glassey, W. V. bind (ver 3.0). bind is distributed as part of the YAeHMOP, extended Hückel molecular orbital package, and is freely available athttp://sourceforge.net/projects/yaehmop/.

(25) Ammeter, J. H.; Bürgi, H.-B.; Thibeault, J. C.; Hoffmann, R. J. Am Chem. Soc. 1978, 100, 3686.

(26) Sellers, H.; Ulman, A.; Shnidman, Y.; Eilers, J. E. J. Am. Chem. Soc. 1993, 115, 9389.
(27) Hall, L. E.; Reimers, J. R.; Hush, N. S.; Silverbrook, K. J. Chem. Phys. 2000, 112, 1510.

(28) Zahid, F.; Paulsson, M.; Polizzi, E.; Ghosh, A. W.; Siddiqui, L.; Datta, S. J. Chem. Phys. 2005, 123, 064707.

(29) Shimazaki, T.; Maruyama, H,.; Asai, Y.; Yamashita, K. J. Chem. Phys. 2005, 123, 164111.

(30) Pantazis, D. A.; McGrady, J. E. J. Am. Chem. Soc. 2006, 12, 4128. JP801926D 